\title{
FACTORES DESENCADENANTES DE LAS COMPLICACIONES EN PACIENTES CON APENDICITIS AGUDA
}

\section{Trigger factors for complications in patients with Acute Appendicitis}

\author{
*Allan Fernando Delcid Morazán, *Aristides Barahona Andrade, *Moran E Barcan Batchvaroff.
}

\section{RESUMEN}

Antecedentes: La apendicitis aguda es la urgencia quirúrgica más frecuente en las emergencias y lleva a serias complicaciones. Su incidencia anual es de 1,33 por 1000 hombres y 0,09 por 1000 mujeres. El diagnóstico temprano, profilaxis antibiótica y terapia postoperatoria correcta, evitan complicaciones. Objetivo: Demostrar los principales factores desencadenantes de las complicaciones en los pacientes con Apendicitis Aguda en el Hospital General del Sur de Junio de 2015 a Febrero de 2016. Pacientes y métodos: Estudio no experimental, transversal, analítico, con universo de 568 pacientes operados por apendicitis aguda, muestra de 300 pacientes, constituida por 179 casos y 121 controles. Se determino un intervalo de confianza de 3.88 , con nivel de confianza de $95 \%$, el valor de precisión 0.6, dando como resultado una muestra de 237 la cual se cambio a 300 por tener más disponibilidad de los expedientes y realizar un estudio más preciso. Resultados: 179 (59\%) de los pacientes eran hombres, $128(70.1 \%)$ tenían entre 1-29 años, el diagnóstico se realizó con: hipersensibilidad en cuadrante inferior derecho del abdomen (98\%), neutrofilia mayor a $70 \%(93 \%)$. No se le aplico profilaxis antibiótica a 204 pacientes (68\%), hubieron apéndicitis complicadas en $59.7 \%$ y no complicadas en $40.3 \%$, infección de la herida quirúrgica en un $10 \%$, y la relación entre uso de profilaxis e infección de herida quirúrgica dio un OR 0.37 Conclusiones: la dificultad en los accesos de salud, la demora en el manejo y tratamiento de los pacientes y la falta de profilaxis antibiótica se vieron como factores desencadenantes. El más importante de estos fue la profilaxis antibiótica ya que su OR de 0.37 nos da un porcentaje de beneficiencia de $63 \%$ para prevenir infección de sitio quirúrgico.

\footnotetext{
* Doctor en medicina y cirugía, egresado de la UNAH.

Dirigir correspondencia a: allanferdelcid@hotmail.com

Recibido: 15 de julio 2016,

Aprobado: 30 de agosto 2016
}

\section{PALABRAS CLAVE}

Apendicitis, Apéndice, Abdomen agudo, Profilaxis antibiótica.

\section{ABSTRACT}

Acute appendicitis is the surgical emergency that is most admitted into emergencies and can lead to serious complications. It has an annual incidence of 1.33 per 1000 men and 0.09 per 1000 women. Early diagnosis, antibiotic prophylaxis and proper postsurgical antibiotic therapy prevent complications. Objective: Demonstrate the principle trigger factors for complication in patients with acute appendicitis in Hospital General del Sur from June 2015 to February 2016. Patients and methods: Non experimental transversal, analytic study, with a universe of 568 patients who underwent an appendicectomy, sample of 300 patients with 179 cases and 121 controls. It was determined a confidence interval of 3.88 with confidence level of $95 \%$, precision value of 0.6 , giving us a sample of 237 which was change to 300 because we had more access to clinical records and assuring a more precise study. Results: The highest percentage occurs in men representing 179 (59\%), people aged 1-29 years accounted $128(70.1 \%)$, antibiotic prophylaxis wasn't applied in 204 patients (68\%), hypersensitivity in right lower quadrant (98\%), neutrophilia greater than $70 \%$ (93\%), complicated appendix (59.7\%) and no complicated acute appendicitis (40.3\%), surgical site infection $(10 \%)$, the relationship between use of prophylaxis and surgical wound infection gives an OR 0.37 Conclusions: the difficulty in access to health, the delay in the management and treatment of patients and lack of antibiotic prophylaxis were as triggers. The most important of them is the antibiotic prophylaxis with an OR of 0.37 and which makes it a protective factor with $63 \%$ of protection for surgical site infection.

\section{KEYWORDS}

Appendicitis, Appendix, Acute Abdomen, Anti- 
biotic prophylaxis.

\section{INTRODUCCIÓN}

La apendicitis aguda es la enfermedad intrabdominal de urgencia que se presenta con mayor frecuencia y es potencialmente mortal en caso de no recibir asistencia médica oportuna. ${ }^{(1)} \mathrm{El}$ tratamiento quirúrgico es uno de los mayores adelantos en salud pública de los últimos 150 años. Con incidencia anual de 1,33 casos por 1000 hombres y 0,09 casos por 1000 mujeres, con un pico máximo entre los 10 y 30 años. ${ }^{(2)}$ En el mundo, la apendicitis perforada es la principal causa quirúrgica de muerte y su pronóstico es más favorable cuando se interviene precozmente. ${ }^{(3)}$

En la práctica clínica, la apendicetomía es realizada por el cirujano, cuyo diagnóstico puede apoyarse en exploraciones complementarias (laboratorio, ecografía y tomografía axial computarizada), pero es básicamente clínico, la historia clínica enfocada en la evolución del dolor y los síntomas asociados así como los hallazgos obtenidos durante el examen físico son aún las piedras angulares del diagnóstico. ${ }^{(4)}$ La población que se interviene por esta enfermedad es mayoritariamente joven y con escasa comorbilidad, también puede presentarse en pacientes de edad avanzada con enfermedades asociadas de riesgo. ${ }^{(3)}$ Cuando se establece el diagnóstico de apendicitis aguda lo recomendado es el tratamiento quirúrgico oportuno. El retraso del mismo por administración de líquidos parenterales y antibióticos se ha visto utilizado en pacientes que lucen tóxicos, niños o mayores con la finalidad de estabilizarlos previa intervención quirúrgica y garantizando un mejor resultado. ${ }^{(5)} \mathrm{La}$ prueba de oro para el diagnostico definitivo de apendicitis es el hallazgo histopatológico del apéndice resecada, el cual se realiza con biopsia; sin embargo en el medio dicho estudio no se encuentra disponible de forma rutinaria por ello el diagnostico de apendicitis se fundamenta en la experiencia clínica del médico tratante. ${ }^{(6)} \mathrm{Se}$ encuentra en esta patología contaminación del sitio quirúrgico por múltiples microorganismos, las heridas contaminadas se asocian a un aumento de la incidencia de complicaciones postquirúrgicas. $^{(7)}$

Se han hecho estudios en Honduras de Apendicitis Aguda en el IHSS que indican que la apen- dicetomía laparoscópica se puede realizar de forma efectiva y segura en los cuadros de Apendicitis aguda. La realización de la técnica y el tiempo quirúrgico total están relacionados con la experiencia del cirujano. ${ }^{(8)}$ a su vez otro estudio indica que el diagnóstico de apendicitis es clínico y que la mejor herramienta es el diagnóstico es la valoración exhaustiva por un cirujano de experiencia. La sobre utilización de estudios radiológicos se debe a su indicación por los médicos de atención primaria, cuando lo apropiado es primero realizar la interconsulta al cirujano. No olvidar que en ocasiones el diagnóstico definitivo solo podrá realizarse en sala de operaciones o en el departamento de patología. ${ }^{(9)}$ no se encontró otros estudios recientes, similares sobre dicha patología, ni estudios con una muestra tan significativa y grande.

El propósito del estudio fue demostrar los principales desencadenantes de las complicaciones en los pacientes con Apendicitis Aguda en el Hospital General del Sur de Junio de 2015 a Febrero de 2016.

\section{PACIENTES Y MÉTODOS}

Estudio no experimental, con diseño transversal y alcance analítico realizado en el Hospital General Del Sur, Choluteca, Choluteca durante el período de junio del 2015 a febrero de 2016. La población estuvo constituida por 568 pacientes operados por apendicitis aguda en dicho periodo, la muestra fueron 300 , constituida por 179 casos y 121 controles. Se utilizo la fórmula para tamaño de muestra donde se determino un intervalo de confianza de 3.88, con nivel de confianza de $95 \%$, P siendo el valor de precisión 0.6 , dando como resultado una muestra de 237 la cual se cambio a 300 por tener más disponibilidad de los expedientes y realizar un todos los pacientes que ingresaron con diagnostico de apendicitis y tuvieron complicación y control todos los pacientes que ingresaron con diagnóstico de apendicitis y no tuvieron complicación.

Criterios de inclusión: Se incluyo a todos los pacientes a quienes se les realizó apendicectomía por apendicitis aguda, de todas las edades y se excluyeron pacientes embarazadas, expedientes incompletos y pacientes que ingresaron con diagnóstico de apendicitis aguda y su diagnóstico se descarto por especialista en cirugía. 
La información fue obtenida directamente de los expedientes mediante un instrumento validado mediante prueba piloto; que contenía datos sociodemográficos, factores relacionado con demora en el tratamiento, hallazgos clínicos, conducta quirúrgica y hallazgos transoperatorios y postoperatorios.

Se utilizó la Escala de Alvarado la cual es una herramienta diagnóstica útil para apendicitis aguda, que considera ocho características principales extraídas del cuadro clínico: migración del dolor (a cuadrante inferior derecho), anorexia y/o cetonuria, náuseas y/o vómitos, sensibilidad en cuadrante inferior derecho (del inglés tenderness), rebote, elevación de la temperatura, leucocitosis mayor o igual a 10,500, desviación a la izquierda de neutrófilos (del inglés shift to the left). Se le asignó un punto a cada característica encontrada, exceptuando sensibilidad en cuadrante inferior derecho y leucocitosis a las que se les asignó 2 puntos para cada uno totalizando 10 puntos. Si la sumatoria es de 7 o más puntos el paciente requiere cirugía, ya que se considera cursa con apendicitis aguda, con 5 y 6 puntos el paciente cursa con una probable apendicitis y se requerirá de valoraciones seriadas tanto clínica como de laboratorio así como de algunos estudios por imágenes (USG, TAC). Si el puntaje es de 1 a 4 puntos existe una muy baja pro babilidad de apendicitis. ${ }^{(10)}$ Los expedientes se escogieron al azar y se revisaron en el archivo del Hospital. Se realizó la digitalización y análisis de resultados con el software Epi Info 7.2. Para las variables descriptivas se calcularon frecuencias y porcentajes, y para los factores asociados a complicaciones se calculó el Odds Ratio.

Aspectos Éticos se obtuvo autorización del director ejecutivo de dicho hospital. Los autores declaramos que no existe conflicto de interés.

\section{RESULTADOS}

De los 300 pacientes que se investigaron, 179 $(59.7 \%)$ eran del sexo masculino. $98(32.7 \%)$ tenían entre 20-29 años, 226 (75.3\%) eran alfabetas. Procedían de municipios del Departamento de Choluteca (49\%) y de la ciudad de Choluteca (39\%). Ver tabla No. 1.
Tabla 1: Características socio demográficas de los pacientes, $n=300$

\begin{tabular}{lrr}
\hline Variable & $\mathbf{N}$ & $\%$ \\
\hline Edad & 25 & $8.4 \%$ \\
$1-9$ & 87 & $29 \%$ \\
$10-19$ & 98 & $32.7 \%$ \\
$20-29$ & 48 & $16 \%$ \\
$30-39$ & 22 & $7.3 \%$ \\
$40-49$ & 16 & $5.3 \%$ \\
$50-59$ & 4 & $1.3 \%$ \\
$>60$ & & \\
Sexo & 121 & $40.3 \%$ \\
Femenino & 179 & $59.7 \%$ \\
Masculino & & \\
Nivel de educación & 226 & $75.3 \%$ \\
Alfabeta & 20 & $6.7 \%$ \\
Analfabeta & 54 & $18 \%$ \\
No consignado & & \\
Procedencia & 146 & $49 \%$ \\
Municipios de Choluteca & 117 & $39 \%$ \\
Choluteca, Choluteca & 27 & $8.9 \%$ \\
Valle & 7 & $2.3 \%$ \\
El Paraíso & 3 & $0.9 \%$ \\
\hline Francisco Morazán &
\end{tabular}

En promedio el tiempo entre el inicio de los síntomas y la llegada de los pacientes al hospital fue $13-24$ horas en $40.7 \%$, el tiempo que tardó en ingresar un paciente desde su llegada fue de 2-3 horas en $36 \%$ de los casos y el tiempo desde el ingreso del paciente hasta la cirugía fue 2-3 horas en 122 (42\%). A 204 (68\%) de los pacientes, no se le aplicó profilaxis antibiótica. En 229 (76.4\%) pacientes, se realizó incisión de Mcburney, y a $41(13.6 \%)$ se les colocó dreno tubular subcutáneo. Ver tabla No. 2.

Tabla No. 2: Características de tratamiento médico-quirúrgico brindado a pacientes, $n=300$

\begin{tabular}{lrr}
\hline Variable & $\mathbf{N}$ & \multicolumn{1}{c}{$\%$} \\
\hline $\begin{array}{l}\text { Tiempo inicio de síntomas hasta } \\
\text { que llega al hospital (en horas) }\end{array}$ & & \\
< o igual 6 & 23 & $7.7 \%$ \\
$7-12$ & 64 & $21.3 \%$ \\
13-24 & 122 & $40.7 \%$ \\
$25-48$ & 52 & $17.3 \%$ \\
$>$ >48 & 39 & $13 \%$ \\
Tiempo transcurrido desde & & \\
Menor o igual a 1 & 104 & $34.7 \%$ \\
2-3 & 108 & $36 \%$ \\
$4-5$ & 58 & $19.3 \%$ \\
Más de 6 & 30 & $10 \%$
\end{tabular}




\begin{tabular}{|c|c|c|}
\hline \multicolumn{3}{|l|}{$\begin{array}{l}\text { Tiempo transcurrido entre } \\
\text { ingreso y cirugía }\end{array}$} \\
\hline Menor o igual a 1 & 43 & $14.3 \%$ \\
\hline $2-3$ & 126 & $42 \%$ \\
\hline $4-5$ & 68 & $22.7 \%$ \\
\hline Más de 6 & 63 & $21 \%$ \\
\hline \multicolumn{3}{|l|}{ Uso de profilaxis antibiótica } \\
\hline $\mathrm{Si}$ & 96 & $32 \%$ \\
\hline \multicolumn{3}{|l|}{ Tipo de abordaje } \\
\hline Incisión Mcburney & 229 & $76.4 \%$ \\
\hline Incisión línea media-infraumbilical & 39 & $13 \%$ \\
\hline Incisión Rocky-Davis & 4 & $1.3 \%$ \\
\hline Incisión xifo-púbica & 3 & $1 \%$ \\
\hline No se consigno & 25 & $8.3 \%$ \\
\hline \multicolumn{3}{|l|}{$\begin{array}{l}\text { Colocación de dreno tubular } \\
\text { subcutáneo }\end{array}$} \\
\hline No & 259 & $86.4 \%$ \\
\hline SI & 41 & $13.6 \%$ \\
\hline \multicolumn{3}{|c|}{$\begin{array}{l}\text { Entre los hallazgos en la escala de Alvarado } \\
\text { hipersensibilidad en cuadrante inferior derech } \\
\text { del abdomen ( } 98 \%) \text {, leucocitosis }(93 \%) \text { y neu } \\
\text { trofilia mayor a } 70 \%(91 \%) .51 .3 \% \text { obtuvieror } \\
\text { entre } 9-10 \text { puntos y } 93 \% \text { de los casos tuvo } 7 \text { c } \\
\text { más puntos en dicha escala. Ver tabla No. } 3\end{array}$} \\
\hline \multicolumn{3}{|c|}{$\begin{array}{l}\text { Tabla No. 3: Escala de Alvarado para el diag } \\
\text { nóstico y tratamiento de los pacientes }\end{array}$} \\
\hline Variable & $\mathbf{N}$ & $\%$ \\
\hline $\begin{array}{l}\text { Hipersensibilidad en cuadrante } \\
\text { inferior derecho del abdomen }\end{array}$ & 294 & $98 \%$ \\
\hline Leucocitosis & 279 & $93 \%$ \\
\hline Neutrofilia mayor a $70 \%$ & 273 & $91 \%$ \\
\hline Nauseas o vómitos & 244 & $81.3 \%$ \\
\hline Signo de Blumberg positivo & 243 & $81 \%$ \\
\hline Anorexia & 209 & $69.7 \%$ \\
\hline Temperatura mayor a $37.2 \mathrm{C}$ & 188 & $62.7 \%$ \\
\hline Migración del dolor & 181 & $60.3 \%$ \\
\hline \multicolumn{3}{|l|}{$\begin{array}{l}\text { Puntaje en escala diagnostica } \\
\text { de Alvarado }\end{array}$} \\
\hline$<4$ & 6 & $2 \%$ \\
\hline $5-6$ & 15 & $5 \%$ \\
\hline $7-8$ & 125 & $41.7 \%$ \\
\hline $9-10$ & 154 & $51.3 \%$ \\
\hline
\end{tabular}

De los 300 pacientes estudiados; 121 (40.3\%) no sufrió complicación y 179 (59.7\%) sufrió complicación. Los hallazgos intraoperatorios fueron apéndices perforadas $98(32.7 \%)$ de los casos y apéndice gangrenosa en 81 (27\%), lo que es considerado apendicitis complicada (Ver Tabla
No. 4), 30 (10\%) pacientes sufrieron infección de la sitio quirúrgico, de los cuales 25 no recibió profilaxis antibiótica y $5 \mathrm{si}$, de los 270 restantes 176 no recibió profilaxis y 94 si recibió profilaxis dando como resultado un OR de 0.37.

Tabla No. 4: Factores desencadenantes de complicación en pacientes con apendicitis

\begin{tabular}{lccccc}
\hline Variable & \multicolumn{5}{c}{ Complicación } \\
\hline Edad & Si & $\%$ & N & $\%$ & \\
1 a 29 & 128 & $71.5 \%$ & 82 & $67.9 \%$ & 0.83 \\
30 o mas & 51 & 28.5 & 39 & $32.1 \%$ & \\
Sexo & & & & & \\
Femenino & 75 & $41.9 \%$ & 46 & $38 \%$ & 0.85 \\
Masculino & 104 & $58.1 \%$ & 75 & $62 \%$ & \\
Nivel de & & & & & \\
educación & & & & & \\
Alfabeta & 161 & $89.9 \%$ & 100 & $82.6 \%$ & 0.44 \\
Analfabeta & 15 & $8.3 \%$ & 21 & $17.4 \%$ & \\
**Tratamiento & & & & & \\
previo a llegada & & & & & \\
al Hospital & & & & & \\
No & 118 & $65.9 \%$ & 80 & $66.1 \%$ & 1.00 \\
$\quad$ Si & 61 & $34.5 \%$ & 41 & $33.9 \%$ &
\end{tabular}

Tiempo de inicio de síntomas hasta que llega al hospital (horas) Menor o igual a 24 horas

Mayor a 24 horas

$107 \quad 59.8 \% \quad 102 \quad 67.6 \%$ $\begin{array}{llll}72 & 40.2 \% & 49 & 32.4 \%\end{array}$

1.4

Tiempo entre llegada al Hospital ingreso (horas)

Menor o igual a 1 Mayor a 1

$\begin{array}{llll}60 & 33.7 \% & 44 & 36.4 \%\end{array}$ 1.13

Tiempo transcurrido entre ingreso y cirugía (horas) Menor o igual a 1 Mayor a 1 $119 \quad 66.3 \% \quad 77 \quad 63.6 \%$

Procedencia

Fuera de Ciudad de $\begin{array}{lllll}\text { Choluteca, Choluteca } 64 & 35.8 \% & 53 & 43.8 \% & 0.58\end{array}$ Choluteca

***Terapia Antibió$115 \quad 62.2 \% \quad 68 \quad 56.2 \%$

tica Utilizada

Monoterapia

Doble Terapia

$\begin{array}{cccc}1 & 0.6 \% & 90 & 74.4 \% \\ 159 & 88.8 \% & 31 & 25.6 \% \\ 19 & 10.6 \% & - & -\end{array}$

*OR: Odds Ratio, factores protectores deberán ser menor a 1.

** Evalúa la conducta del especialista en cirugía sobre la terapia antibiótica postoperatoria.

*** Indica tratamiento empírico o medico previo a la llegada del paciente al hospital. 


\section{DISCUSIÓN}

La apendicitis aguda es una de las patologías quirúrgicas más frecuente en nuestro medio y a nivel mundial, los resultados encontrados en este estudio indican que la presentación típica con el cuadro de apendicitis se dio en el rango de edad de $20-29$ años en $32.7 \%$ y el segundo rango fue de $10-19$ años $29 \%$, resultados que concuerdan con la estadística que presentan otros estudios que resaltan que dicha enfermedad se presenta en su mayoría entre la segunda y tercer década de la vida. ${ }^{(11)} \mathrm{E} n$ cuanto al sexo, se presentó mas en el sexo masculino en $59.7 \%$, dicho dato es igual al resultado en un estudio realizado en Colombia presentado en el 7th Annual Academic Surgical Congress donde se diagnosticaron $59.7 \%$ sujetos del sexo masculino. ${ }^{(12)}$ Se presentó un alto nivel de alfabetismo $75.4 \%$, entre las personas que fueron intervenidas quirúrgicamente y ser alfabeta resultó ser un factor protector para complicaciones con un OR de 0.44.

La cobertura del Hospital General Del Sur para pacientes con esta patología es amplia y pudimos observar que aunque la mayoría de los pacientes procedían de municipios de Choluteca $(49 \%)$ y de la ciudad de Choluteca (39\%) también se recibieron pacientes procedentes de Valle en un $8.9 \%$ y departamentos que no corresponden a la región sur del país como El Paraíso y Francisco Morazán representando estos 2.3\% y $0.9 \%$ respectivamente. Es notable por esto que pacientes de sitios alejados de Choluteca necesitan de atención hospitalaria y su opción más cercana es el Hospital Regional del Sur.

La desigualdad y dificultad en el acceso a los servicios de salud se evidenció en los rangos de tiempo que transcurren entre el inicio de los síntomas y la llegada del paciente al hospital pudimos observar que en un $40.7 \%$ el rango fue de $13-24$ horas, agregándole a esto el tiempo transcurrido desde la llegada al hospital hasta el momento que se ingresa que fue de $2-3$ horas en $36 \%$ y el tiempo desde que se ingresa y es llevado a cirugía tiene un rango predominante de 2-3 horas representado por un $42 \%$, considerando estos números estimamos un tiempo aproximado de 17-30 horas desde que inician los síntomas hasta que es intervenido quirúrgicamente, hecho que hay que tomarle importancia ya que hay estudios especializados que establecen que a partir de las 6 horas desde que inician los síntomas el apéndice puede perforase. Lo común es que a 16 horas de iniciada el cuadro se presente algun tipo de complicación en un $35 \%$ y después de 32 horas en un $75 \%$ de las apendicitis son casos complicados. ${ }^{(13)}$ A su vez esto se puede relacionar con que los hallazgos intraoperatorios más frecuentes fueron apéndices perforadas las cuales se presentaronZen $32.7 \%$ de los casos y apéndice gangrenosa en $27 \%$, todas con un tiempo de evolución considerable.

Además vale la pena recalcar que dentro de los resultados se evidenció que el $68 \%$ de los pacientes no recibió profilaxis antibiótica, en su mayoría $(55.4 \%)$ porque no se indicó en ordenes médicas y en menor medida fue indicado en ordenes medicas pero no cumplido (12.3\%), lo cual revela que los pacientes permanecen dentro del Hospital sin ninguna dosis de terapia antibiótica profiláctica que cubra anaerobios y gram negativos, en contraposición la terapia de soluciones electrolíticas por vía parenteral se inicia en un $97.3 \%$, la cual se considera de importancia para la preparación preoperatoria. ${ }^{(14)}$ La efectividad del antibiótico profiláctico en apendicitis aguda está sostenida por un metaanálisis de 45 ensayos donde se notó una reducción significativa en la infección del sitio quirúrgico ( $5 \%$ vs. $11 \%$ ) con un OR de $0.37 .^{(15)}$

De los 300 casos estudiados 30 presentaron infecciones de sitio quirúrgico, lo que corresponde al $10 \%$ del total. La ausencia de la profilaxis antibiótica se relacionó directamente con la infección de sitio quirúrgico como complicación postoperatoria ya que 25 de estos casos no contaron con esta profilaxis $(93.4 \%)$ y solo 5 de los pacientes que recibieron una dosis de antibiótico previa cirugía presentaron esta complicación. Se obtuvo un OR de 0.37 , lo cual evidencia que el beneficio de recibir profilaxis antibiótica previa cirugía otorga un $63 \%$ menos de riesgo de sufrir una infección de sitio quirúrgico, evidenciando a su vez el mismo OR del metaanálisis antes descrito.

De los 300 casos estudiados, 30 sujetos presentaron infección del sitio quirúrgico representando el $10 \%$ de los casos y siendo la principal complicación postoperatoria presentada, al igual como sucedió en un estudio del Hospital de Valdivia, en el cual la complicación postoperatoria más frecuente fue la infección del sitio quirúrgica con 24 pacientes. ${ }^{(16)}$

El dolor abdominal en el cuadrante inferior derecho del abdomen, la anorexia y las nauseas o vómitos son los hallazgos clásicos en un caso de apendicitis aguda. ${ }^{(17)}$ Pudimos observar que los hallazgos clínicos predominantes fueron la 
Hipersensibilidad en cuadrante inferior derecho del abdomen $98 \%$, neutrofilia mayor a $70 \%$ (93\%) y las nauseas o vómitos $91 \%$. Los demás signos y síntomas se presentaron en más del $60 \%$. En general se acepta un puntaje de Alvarado mayor a 7 como un criterio para apendicetomía y como pudimos observar la mayoría de los pacientes tenían una puntuación de 7 o más, observándose en un $51.33 \%$ de $9-10$ puntos y $41.67 \%$ tenían de $7-8$ puntos. Los demás rangos de puntaje representaron juntos solamente un $7 \%$ del total. Esto nos demuestra que la Escala de Alvarado es una herramienta útil para el diagnóstico de apendicitis aguda ya que de los 300 pacientes con apendicitis aguda se encontró criterio quirúrgico por Alvarado en 279 pacientes, lo que representa un $93 \%$. $^{(18)}$

En cuanto al abordaje quirúrgico, se encontró que la incisión de Mcburney fue la más utilizada (76.4\%), igual a lo descrito por cirujano del Hospital Británico de Buenos Aires que a pesar de que existen múltiples tipos de incisiones, la más empleada es la incisión de Mcburney. Aunque se puede utilizar la incisión infraumbilical ya que brinda un campo suficiente para la exploración abdominal. Hoy en día hay evidencia científica por dos meta análisis que recomiendan un abordaje laparoscópico, ya que se muestra un descenso en la estadía hospitalaria (con una diferencia media de 0.5 a 0.6) y una disminución en el riesgo de infección de la herida quirúrgica de 1 a $2 \%{ }^{(17)}$ En el hospital General del Sur no se realiza abordaje laparoscópico de rutina ya que no se cuenta con suficientes especialistas y equipo para tomar esta conducta ya que en las guardias solo hay un cirujano y solo hay una torre de laparoscopia.

La apendicetomía por apendicitis es la operación de urgencia que se practica más a menudo en el Hospital General del Sur, Choluteca, donde se reportaron 568 casos de apendicitis aguda en él año 2015. El estudio de los efectos adversos de la apendicitis tiene especial relevancia por el impacto que ocasionan en la salud de los pacientes y por la repercusión en el gasto social y del sistema de salud local y nacional. Además, es un instrumento de evaluación de la calidad asistencial. Se acepta que aproximadamente una tercera parte de las complicaciones son atribuibles a deficiencias en la atención sanitaria. $^{(19)}$

\section{Conclusiones}

Dentro de los factores desencadenantes para complicaciónes de la apendicitis esta la dificultad en los accesos de salud, el Hospital General del Sur tiene una cobertura amplia para este tipo de pacientes que no solo incluye la zona sur del país. Hay demora en el tratamiento de los pacientes con apendicitis aguda ya que la demanda quirúrgica es grande y se debe brindar capacitación y actualización al personal que ingresa este tipo de pacientes para el diagnostico y aplicación de profilaxis anfibiótica y así evitar complicaciones postoperatorias, y beneficiar al paciente y a su vez al hospital por la reducción de gastos por complicaciones y estadías prolongadas.

\section{Recomendaciones}

Se recomienda al Gobierno de la República de Honduras y a la Secretaria de Salud de Honduras contratar más cirujanos, médicos generales y personal de enfermería para poder manejar oportunamente este tipo de patologías. A la Dirección y al Departamento de Cirugía del Hospital General del Sur se recomienda desarrollar un protocolo de manejo para pacientes con apendicitis aguda para mejorar la calidad del manejo de los pacientes.

\section{Agradecimientos}

Agradecemos al Director y al personal de Archivo y Estadística del Hospital General del Sur por habernos permitido la revisión de los expedientes, a los especialistas del Departamento de Cirugía por guiarnos y enseñarnos sobre el arte de la cirugía, al Doctor Aristides Barahona Gómez por su asesoría temática, a los docentes de la Facultad Ciencias Medicas por despejar nuestras dudas con respecto a esta investigación.

\section{BIBLIOGRAFÍA}

1. Suárez LDQ, Milagros D, Pelayo I, Caridad D. La escala de Alvarado como recurso clínico para el diagnóstico de la apendicitis aguda Alvarado score used as a clinical resource for diagnosing acute apendicitis. 2015; 54(2):121-8.
2. Godoy, Alí Itriago, Diego Machado, Tomás Nieves, Anne Ramírez J.fff Apendicectomía Abierta Vs Apendicectomía Por Laparoscopia. Experiencia Del Servicio De Cirugía Uno. Hospital Vargas De Caracas. Rev Venez Cir. 2006; 71-7. 
3. John Maa, Kimberly S. Kirkwood. El Apendice. En: Courtney M. Townsend, Jr., R. Daniel Beauchamp, B. Mark Evers, Kenneth L. Mattox M. Sabiston Tratado de Cirugía Edición. España: Elsevier; 2013. 1333- 1347.

4. González JF. Apendicitis Aguda Revisión Bibliográfica. Med Leg Costa Rica.

2012;29(1):83-90.

5. Tagle DL, Ferrer MH, Arias TS, Hernández TS, Dupeyrón OV. Infección de la herida quirúrgica. Aspectos epidemiológicos. Rev Cuba Med Mil. 2007; 36(2):1-11.

6. Jose' Manuel Aranda-Narvaez, María Custodia Montiel-Casado, Antonio Jesús González-Sánchez, Carolina Jimenez-Mazure, Marta Valle-Carbajo BS-P y JS-S. Empleo, eficacia y repercusión clínica del apoyo radiológico al diagnóstico de la apendicitis aguda. Cir Esp. 2013;91(9):574-8.

7. Vasen W. Infección del Sitio Quirúrgico. Cir Dig. 2009;1:1-10.

8. Marcial Zúniga Ras. Uso De Técnica Laparoscopica En Pacientes Con Apendicitis Aguda Operados En El Hospital De Especialidades IHSS. Rev Medica de Los Post grados Med UNAH. 2007; 10(1):156-160.

9. Barahona Jl, Rittenhouse Dw. Apendicitis Aguda ¿Ser O No Ser? Revisión Bibliográfica Pediátrica. Rev Med Hondur. 2009; 77(3):118-21.

10. Velázquez JD, Godínez C, Vázquez M. Evaluación prospectiva de la Escala de Alvarado en el diagnóstico de apendicitis aguda Prospective evaluation of the Alvarado Scale in the diagnosis of acute appendicitis. Cir Gen. 2010;32:17-23.

11. Crusellas O, Comas J, Oscar V, Benerroch G. Manejo y tratamiento de la apendicitis aguda. Atención Urgent [Internet]. 2008[citado 09 de mayo 2016];5. Disponible en: www.jano.es/ficheros/sumarios/1/0/1682/ 29/002900 33-LR.pdf.

12. Sanabria A, Dominguez L, Vega $V$, Osorio $C$, Serna A, Bermudez C. Tiempo de evolución de la apendicitis y riesgo de perforación. Rev Colomb [Internet]. 2013[citado 10 de mayo 2016];24-30. Available from: http:// www.scielo.org.co/scielo.php?script= sci_arttext\&pid=S2011-758220130001000 03.

13. Dubon M, Ortiz A. Apendicitis Aguda, Su Diagnostico Y Tratamiento. Rev la Fac Med dela UNAM [Internet]. 2014[citado 12 de mayo 2016];57(4):51-7. Disponible en: http://www.medigraphiccom/pdfs/facmed/ un-2014/un144h.pdf.

14. Enrique Gómez Bravo Topete, Alberto Ernesto Hardy Pérez LEHG de A. Apendicitis Aguda. Med Urgencias Prim Niv Aten. 2004;1-5.

15. Ronald F Martin M. Acute appendicitis in adults: Clinical manifestations and differential diagnosis. UpToDate [Internet]. 2016[citado 13 de mayo 2016];1-9. Disponible en: https://www.uptodate.com/contents/acutea ppendicitisinchildrenmanagement?topicKey $=\mathrm{EM} \% 2 \mathrm{~F} 6481 \&$ elapsedTimeMs=8\&sour $c e=$ see_link\&view $=$ print\&d.

16. Carlos Cárcamo, Elisa Rivera AS. Apendicitis aguda en el Hospital Clínico Regional de Valdivia. Cuad Cir. 2002; 16:11-5.

17. Castagneto GH. Patología quirúrgica del apéndice cecal. Hosp Británico Buenos Aires [Internet]. 2010[citado 13 de mayo 2016];3:1-11. Disponible en: http://www.sa cd.org.ar/tcuatro.pdf.

18. Reyes-García N, Zaldívar-Ramírez FR, CruzMartínez R, Sandoval-Martínez MD, Gutiérrez-Banda CA, Athié-Gutiérrez C. Precisión diagnóstica de la escala RIPASA para el diagnóstico de apendicitis aguda: análisis comparativo con la escala de Alvarado modificada. Cir Gen [Internet]. 2012[citado 30 de mayo 2016];34(2):101-6. Available from: http://www.scielo.org.mx/scielo. php?script=s ci_arttext\&pid=S1405-00992012000200002\& Ing=es\&nrm=iso\&t lng=es.

19. Aguilo Javier, Peiro Salvador, Munoz Carmen, García del Cano Julian, Garay Miguel, Viciano Vicente, et al. Efectos adversos en la cirugia de la apendicitis aguda. Cir Esp [Internet]. 2005[Citado 29 de mayo 2016];78(5):54-9. Disponible en: https://apps.webofknowledge.com/full_re cord.do? product=UA\&search mode $=$ Gene ralSearch\&qid=1\&SID=X1VbNJbsC 2XLku Reznp\&page $=1 \& d o c=8$. 